\title{
Der erkenntnistheoretische Pazifismus: Programmschrift für ein philosophisches Plädoyer gegen unsere Kriege
}

\author{
Olaf L. Müller*
}

\begin{abstract}
I wish to propose a new doctrine called epistemological pacifism. According to the doctrine, our objective knowledge concerning hard facts about a given war and its context is far too poor to justify entering that war. Our best and most informative accounts of any pre-war situation are value-laden; the same is true of counterfactual claims about any event during, or after, war. Here we have three new types of what has been discussed under the label of fact/value entanglement. Realizing this helps us understand why pacifists and their opponents never agree about so-called facts. Both parties bring to bear different values in their descriptions. Although this is legitimate for both sides, the values of the pacifist are more attractive than those of the bellicist. The recent war in Mali is a case in point, as I'll sketch at the end of the paper.
\end{abstract}

Keywords: Pacifism, bellum iustum, epistemology, Kosovo war, Mali

Pazifismus, bellum iustum, Erkenntnistheorie, Kosovokrieg, Mali.

\section{Vorbemerkung: Wagnisse eines programmatischen Unterfangens}

1 ls mich die Anfrage ereilte, ob ich für diese Heftausgabe einen Beitrag über erkenntnistheoretischen Pazifismus beisteuern möchte, sah ich darin eine willkommene Gelegenheit, meine philosophischen Argumente gegen die letzten Interventionskriege des Westens programmatisch auf den Punkt zu bringen. Was ich bislang gegen unsere Interventionen z.B. im Kosovo oder in Afghanistan vorgebracht habe, folgt zwar einer einheitlichen Methode und läuft jedesmal auf dasselbe pazifistische Ergebnis hinaus - aber Methode und Ergebnis drohen hinter den unzähligen Details $\mathrm{zu}$ verschwinden, die fürs jeweilige Argument erforderlich sind. Deshalb ist es zuweilen unabdingbar, ein, zwei Schritte zurückzutreten und den Wald zu betrachten, nicht die Bäume. Das möchte ich in meinem Beitrag zum ersten Mal versuchen.

Der Versuch ist riskant. Ich könnte bei Leserin und Leser in den Verdacht geraten, mit allzu groben Pinselstrichen zu argumentieren. Daher möchte ich vorausschicken, dass ich viele der hier nur skizzierten Überlegungen anderswo akribisch ausgearbeitet habe. ${ }^{1}$ Ich werde im bevorstehenden Gedankengang jeweils an Ort und Stelle knapp auf die Veröffentlichungen dieser Details verweisen.

\section{Erkenntnistheorie und Pazifismus - zwei kühne Begriffe}

Sabine Jaberg hat meine bisherigen Arbeiten zu Krieg und Frieden als erkenntnistheoretischen Pazifismus charakterisiert. Diese beiden kühnen Worte treffen verblüffend gut, worauf es mir

* Olaf Müller leistete seinen Wehrdienst in den Jahren 1985/86. Während seines Philosophie- und Mathematikstudiums in Göttingen verweigerte er nachträglich den Dienst an der Waffe. Seitdem er die zugehörige Gewissensprüfung überstanden hat, arbeitet er an der philosophischen Frage, wie der Pazifismus am besten formuliert werden sollte. Er ist seit 2003 Professor für Wissenschaftsphilosophie an der Humboldt-Universität zu Berlin.

1 Siehe die Sammlung von ethischen Argumenten und Fallstudien in O.M. [PP]. ankommt. Das erste Wort charakterisiert meine Methode, das zweite mein Ergebnis.

Zum ersten: Während der letzten Jahrzehnte sind die erklärten Pazifisten in der akademischen Philosophie Deutschlands immer weniger geworden. Gegen diesen Trend setze ich in meinen Überlegungen zum Krieg dezidiert die Mittel der gegenwärtigen Philosophie ein - genauer gesagt, die Mittel der Erkenntnistheorie. Das ist eine altehrwürdige Disziplin, die uns ständig daran erinnert, wie wenig wir wissen. Ich weiß, dass ich nichts weiß, so hat es Sokrates (laut Platon) in seiner Verteidigungsrede vor dem Athener Gericht zugespitzt. In aller Allgemeinheit ist und war das übertrieben. ${ }^{2}$ Doch beim Thema Krieg oder Frieden, ja schon bei der Frage nach den Fakten im Vorfeld und Umfeld von Krieg, trifft das Denkmotto des Sokrates genau ins Schwarze. Wann immer wir über Krieg oder Frieden entscheiden sollen, wissen wir viel zu wenig: erstens zuwenig, um einen verantwortungsvollen Eintritt in den Krieg rechtfertigen zu können; zweitens zuwenig, um einen verantwortungsvollen Kriegsverzicht rechtfertigen zu können. Man mag fragen: Läuft der erkenntnistheoretische Anteil in meinen Überlegungen nur darauf hinaus, an eine legendäre Binsenweisheit der beginnenden abendländischen Philosophie zu erinnern? Nein; er reicht bis in die moderne Erkenntnistheorie der Moral (die im Jargon der Philosophen oft als Metaethik bezeichnet wird). Kurzum, meine Methode ist erkenntnistheoretisch im weitesten Sinn. (Mehr dazu in den Abschnitten 4 und 5).

Der zweite Begriff heißt Pazifismus und ist nicht minder gewagt. Man kann lange darüber sinnieren, was dieser Begriff genau bedeutet und was nicht. Johan Galtung hat mir bereits im Jahr 2002 davon abgeraten, von Pazifismus zu reden - der Begriff sei mittlerweile mit soviel schlechtem Beigeschmack versehen, dass er für die ernsthafte Diskussion nichts mehr tauge. Ich finde das zu defaitistisch. Lassen Sie mich kurz andeuten, wie ich den Begriff verstehe: Pazifist oder Pazi-

2 So habe ich anderswo gezeigt, dass die Welt keine Computersimulation à la Matrix sein kann, siehe O.M. [HPAS] 
fistin ist, wer in der politischen oder ethischen Diskussion über Krieg und Frieden bei jedem einzelnen Beispiel zu dem Ergebnis kommt, dass der diskutierte Kriegseinsatz moralisch falsch ist, falsch war, falsch sein wird, falsch wäre. In diesem Verständnis kommt es (für die Begriffsbestimmung des Pazifismus) nicht darauf an, aus welchen Gründen und mithilfe welcher ethischen Theorie die Pazifisten zu ihrem immer wieder gleichlautenden Ergebnis gelangen. Die Gründe mögen religiös, rigoristisch, gesinnungsethisch, verantwortungsethisch oder erkenntnistheoretisch sein; daraus ergeben sich nur verschiedene Spielarten von Pazifismus. Ich plädiere für die erkenntnistheoretische Spielart dieser Position, und zu ihren vielen Verwandten will ich hier nichts sagen; sie kommen mir weniger attraktiv vor.

\section{Stellen wir ein Vorurteil vom Kopf auf die Füße}

Pazifisten sind gefühlsduselige Gutmenschen, verantwortungslose Zauderer, irrationale Spinner, Traumtänzer mit Jesuslatschen, dubiose Gesinnungsethiker. Diese Vorurteile bekämpfe ich seit mehr als einem Vierteljahrhundert. Mit rationalen, philosophischen Argumenten versuche ich zu zeigen, dass die Verfechter militärischer Interventionen - die Kriegsbefürworter - weder Realismus noch Verantwortungsethik auf ihrer Seite haben; sie verfehlen also ihren eigenen Anspruch. Weder berufen sie sich (zur Begründung dieses oder jenes Kriegs) ausschließlich auf unbestreitbare Fakten; noch können sie die Folgen militärischer Eingriffe seriös abschätzen. Und also können sie erst recht nicht auf verantwortungsvolle Weise für militärische Einsätze plädieren.

Um es zu überspitzen: Die letzten Kriege des Westens wurden von unfreiwilligen Hasardeuren in Angriff genommen; und mit unseren zukünftigen Kriegen wird es kaum besser stehen. Diese Diagnose resultiert aus zwei verschiedenartigen Gedankengängen, die einander in die Hände spielen. Einerseits ergibt sie sich abstrakt aus erkenntnistheoretischen Überlegungen und andererseits konkret aus Fallstudien über einzelne Kriege. Ich skizziere die beiden Gedankengänge in den nächsten Abschnitten und beginne mit Erkenntnistheorie.

\section{Gegen unser angeblich objektives Wissen über die einschlägigen Fakten im Umfeld von Krieg}

Meine erkenntnistheoretische These lautet: In den allermeisten Fällen wissen wir prinzipiell zu wenig, um die Folgen von Krieg und Kriegsverzicht einander unvoreingenommen oder wertfrei gegenüberzustellen. Das trifft Kriegsbefürworter wie Kriegsgegner gleichermaßen und hat nichts mit mangelnder Sorgfalt zu tun. Was wir wertfrei über einen Krieg wissen können, liegt auf einer niedrigeren Ebene als das, was wir darüber wissen müssen.

Dies ist ein skeptisches Resultat - aber kein Plädoyer dafür, klein beizugeben; kein Plädoyer für Urteilsenthaltung. Im Gegenteil, mein philosophisches Ergebnis hat auch eine positive Seite: Wenn aus erkenntnistheoretischen Gründen keine Chance auf objektive, wertfreie Ermittlung der Fakten besteht, dann bleibt uns nichts anderes übrig, als die Faktenlage im
Lichte unserer besten Werte zu beschreiben. ${ }^{3}$ De facto versuchen das die meisten redlichen Teilnehmer am Streit für und wider Krieg - aber verdeckt; fast immer, ohne es zu bemerken. Es täte dem Diskurs über Krieg gut, wenn allen bewusst wäre, wie wichtig unsere Werthaltungen bereits für jedwede informative Beschreibung der einschlägigen Tatsachen im Vorfeld, Umfeld und Rückblick eines Kriegs sind. Ich sehe es als wichtige Funktion der Philosophie, versteckte Voraussetzungen ans Licht zu bringen; man könnte es Aufklärung nennen. Genau darauf zielt dieser Teil meiner erkenntnistheoretischen Arbeit. Auch Gegnerinnen des Pazifismus können davon profitieren. ${ }^{4}$

Dass sich Werte und Fakten auf unentwirrbare Weise durchdringen, ist keine neue These; sie ist z.B. während der letzten Jahrzehnte von einer Reihe analytischer Philosophen verfochten worden, in der neueren Metaethik. ${ }^{5}$ Doch meine Anwendung der These auf Krieg und Frieden ist neu (soweit ich weiß). Wir tun gut daran, die These nicht einfach aus der Literatur auf Krieg zu übertragen, sondern sie erst einmal durch Blick auf ein allägliches Beispiel zu durchleuchten; dabei gewinnt sie an Kontur und hoffentlich auch an Plausibilität. Das will ich im nächsten Abschnitt vorführen.

\section{Rätselhafte Wenn/dann-Sätze}

Im moralischen Alltag und bei der Rede über Krieg benutzen wir gern ganz bestimmte Wenn/dann-Sätze, die bei näherem Hinsehen rätselhaft wirken. Zum Beispiel sagen wir: "Wenn er sein Portemonnaie nicht vergessen hätte, dann wäre er nicht schwarzgefahren". Einerseits enthält dieser Satz zwei Informationen über Fakten, die sich ohne Mühe objektiv eruieren lassen; erstens ist die betreffende Person schwarzgefahren, zweitens hat sie ihr Portemonnaie vergessen. Andererseits behauptet der Gesamtsatz deutlich mehr als diese zwei Tatsachen; er behauptet einen inneren Zusammenhang. Wie können wir diesen inneren Zusammenhang objektiv feststellen? Über dies Rätsel zerbrechen sich die besten Philosophen seit hundert Jahren den Kopf. ${ }^{6}$

Was müsste man beobachten, welche wissenschaftliche Theorie müsste man aufbieten, welches Experiment anstellen, um derartige Wenn/dann-Sätze objektiv zu begründen? Bedenken Sie, dass der Satz aus meinem Beispiel etwa zur Entschuldigung des Schwarzfahrers herangezogen werden könnte, aus Liebe zu oder Respekt vor dem Erwischten. Offenbar kommt es für die überzeugende Verwendung des Satzes nicht so sehr darauf an, ob der Satz die Tatsachen trifft - sondern darauf, ob er

3 Mehr dazu in O.M. [PmOA], 12. Abschnitt.

4 Die erklärte Befürworterin des Kriegs gegen den Terrorismus Jean Bethke Elshtain ist eine der wenigen Personen, die in dieser Angelegenheit zum selben Ergebnis kommt wie ich; sie spricht von der moralischen Natur der Faktenwelt (Elshtain [JWaT], S. 15/6). Was ich bis hierher gesagt habe, können also auch Gegner des Pazifismus unterschreiben, ohne ihre Position preisgeben zu müssen.

5 Schon lange vorher kursierten ähnliche Thesen, etwa mit Blick auf die Methoden der Geschichtsschreibung, siehe Rickert [KN], S. 82-86 et passim. Ohne ausdrücklichen Bezug darauf erlangte die These in der analytischen Philosophie erneute - metaethische - Aufmerksamkeit; siehe z.B. Williams [ELoP], S. 140-141; Murdoch [IoP], S. 22-23, 42; McDowell [AMRH], S. 21; Putnam [RTH], Kapitel 5-9, insbes. S. 139-141; Putnam [OSED]; Putnam [CoFV]. In den Überlegungen dieser Autoren spielen die sog. dichten ethischen Begriffe („thick ethical concepts“) eine Schlüsselrolle, und natürlich haben diese Gedankengänge auch Kritik herausgefordert, siehe z.B. Millgram [IEB].

6 Viele Details hierzu und zum Folgenden in O.M. [CKK], 3. bis 10. Abschnitt. 
angemessen ist, auch und gerade in seiner impliziten Wertung. Aus diesen und ähnlichen Erwägungen halte ich solche Wenn/dann-Sätze für wertbeladen; sie drücken eine wertbeladene Beschreibung aus. Und falls ich richtig liege, gilt diese erkenntnistheoretische oder metaethische These besonders deutlich für diejenigen Wenn/dann-Sätze, ohne die wir beim Streit über Krieg nicht weit kommen. ${ }^{7}$

Wirkt das auf Sie wie eine eigentümliche akademische Übung aus dem Elfenbeinturm? Verständlich wäre es, aber nicht schlimm. Manchmal muss man in den Elfenbeinturm, um in Ruhe nachzudenken. Und vielleicht ist Ihnen schon aufgefallen, wie viele dieser seltsamen Wenn/dann-Sätze wir im Streit über Krieg und Frieden benutzen. Zum Beispiel hört man oft: Hätten Briten und Amerikaner aufs systematische Bombardement deutscher Städte verzichtet, so wäre der Zweite Weltkrieg in Europa nicht schon im Mai 1945 beendet worden und hätte wesentlich mehr Opfer gefordert als de facto. Lassen Sie diesen Satz einmal in einem ruhigen Moment auf sich wirken und fragen sich: Wie soll er objektiv begründet werden? Nimmt es wunder, dass Anhänger unterschiedlicher Wertsysteme zu ganz entgegengesetzten Urteilen über ihn kommen, selbst wenn sie über sämtliche historischen Einzelfakten einig sind? ${ }^{8}$

Was meine erkenntnistheoretische (metaethische) These bei Kriegsproblemen taugt, muss sich anhand tatsächlicher Fälle erweisen. Damit bin ich bei dem zweiten Gedankengang, auf dem meine pazifistische Diagnose beruht: Es gilt anhand der jüngsten großen Kriege in concreto vorzuführen, wie eine extrem friedliebende Betrachtung kriegerischer Fakten aussieht und warum sie attraktiv ist.

\section{Fallstudien gegen eine gigantische Illusion - das Beispiel Kosovo}

Mein wichtigstes Beispiel ist der Kosovokrieg. ${ }^{9}$ Er hat die deutsche Politik grundlegend verändert: Vor Kosovo war es so gut wie undenkbar, deutsche Soldaten zum Kämpfen in die Welt zu schicken. Das zu ändern, stellte einen gewaltigen Kraftakt der (damals frisch gewählten) rot-grünen Bundesregierung dar. Einige ihrer Protagonisten hielten das noch Jahre später für die wichtigste außenpolitische Leistung ihrer Zeit. Sie waren stolz darauf, dafür gesorgt zu haben, dass Deutschland heute mehr Verantwortung in der Welt übernehmen kann als vor Mauerfall und Wiedervereinigung. ${ }^{10}$

Verantwortung ist ein philosophischer Begriff. Wer ihn einsetzt, muss sich an philosophischen Standards messen lassen. Was das heißt, werde ich nur im Stenogrammstil formulie-

7 Selbstverständlich trifft die These nicht auf alle Wenn/dann-Sätze zu, sondern nur auf diejenigen, in denen kontrafaktisch von menschlichem Handeln die Rede ist - und zwar insbesondere dann, wenn es um das gewalttätige Handeln vieler Menschen geht. Details dazu in O.M. [CKK], 8. bis 10 . Abschnitt.

8 Mehr dazu in O.M. [CKK], 9. Abschnitt.

9 Siehe O.M. [WWSü]. Knapper und polemischer habe ich einen analogen Gedankengang auf den Afghanistankrieg zugespitzt (O.M. [UG]). Die französische Intervention in Mali ist mein jüngstes Beispiel, zu dem ich bislang allerdings nicht mehr als eine argumentative Skizze anbieten kann, s.u. Abschnitt 8.

10 So z.B. Peter Struck in Löwenstein [DIEG]. ren. ${ }^{11}$ Die rot-grüne Bundesregierung fühlte sich verantwortlich dafür, serbische Menschenrechtsverletzungen an Kosovo-Albanern zu verhindern. Das ist für sich genommen eine noble Haltung. Hat sie sich einlösen lassen?

Auf welchem Weg soll man den beanspruchten Kausalzusammenhang objektiv feststellen! Blicken wir kurz auf die Fakten, die unumstritten sind: In den Jahren bis zur NATO-Intervention wurden um die tausend Bürger des Kosovo ermordet. Während der dreimonatigen westlichen Luftangriffe ermordeten Serben insgesamt ca. 10.000 Kosovo-Albaner. Das sind die objektiven Fakten und Zahlen, die keiner bestreitet. ${ }^{12}$ Aber was besagen sie über Verantwortung? Ist der Westen mitverantwortlich für die Massaker, die während seiner Luftschläge am Boden wüteten?

Die Antwort hängt davon ab, was die Serben getan hätten, wenn wir sie nicht angegriffen hätten. Haben die Luftschläge Schlimmeres verhütet? Oder haben sie das Schlimmste ausgelöst? Das kann objektiv kein Mensch wissen. ${ }^{13}$ Wir wissen also nicht, ob wir mitverantwortlich sind für die Ermordung von zehntausend Menschen. Das ist ein fatales Ergebnis - jedenfalls für diejenigen, die im Namen der deutschen Verantwortung Soldaten und Flugzeuge losgeschickt haben, um gegen von Serben verübte Menschenrechtsverletzungen zu Felde zu ziehen. ${ }^{14}$

\section{Pazifismus strengt an}

Nehmen wir einmal an, ich läge mit den skizzierten Thesen richtig; dann hinge der Streit zwischen Kriegsbefürwortern und -gegnern weniger von der verwendeten moralischen Theorie ab, als man meinen könnte. Pazifisten sind keineswegs auf Gesinnungsethik festgelegt. Verantwortungsethiker und Utilitaristen aller couleur müssten sich genauso wie die Anhänger der Lehre vom Gerechten Krieg (bellum iustum) immer mit demselben Problem herumschlagen, mit der Wertbeladenheit der einschlägigen Kriegsbeschreibungen.

Selbst wer dieser Lehre anhängt (die bislang immer von verantwortungsbewussten Kriegsbefürwortern hochgehalten wurde), kann mit guten Gründen zu dem Ergebnis kommen, dass jeder unserer Kriege moralisch falsch war. ${ }^{15}$ Wie das? Lassen Sie es mich anhand zweier Elemente der Lehre vom Gerechten Krieg illustrieren, deren Unterscheidung zwischen legitimen und illegitimen Kriegen auch der Pazifist für seine Kriegskritik nutzen kann. Nehmen wir erstens das Kriterium der Proportionalität (aus dem Kriterienkatalog für einen gerecht geführten Krieg, ius in bello). Pazifistische Anhänger dieses Prinzips stimmen zu, dass ein Krieg nur dann korrekt geführt wird, wenn seine negativen Begleiterscheinungen nicht schlimmer sind als die Übel, die er verhindert - sie behaupten aber für jeden Einzelfall, dass die Kollateralschäden sehr wohl schwerer wiegen als die verhinderten Übel. Diese Haltung kann man immer

11 Für die Details siehe O.M. [WWSü], 7. Abschnitt sowie O.M. [PmOA], 4. Abschnitt.

12 Details und Belege dazu in O.M. [PmOA], Anmerkung 25.

13 Mehr dazu in O.M. [CKK], 10. Abschnitt.

14 Und in diesem Resultat sind die Menschenrechtsverletzungen noch gar nicht mitberücksichtigt, deren sich Kosovo-Albaner nach Ende der Bombardierungen schuldig gemacht haben, etwa die Vertreibung von hunderttausend Roma aus ihren Häusern (so jedenfalls ohne Bezug zum Kosovokrieg FAZ, 22.2.2013, S. 1)

15 Siehe O.M. [PmOA], 3. und 4. Abschnitt. 
einnehmen, ohne objektive Fakten in den Wind zu schlagen: Da verhinderte Übel per definitionem nicht in der Welt vorkommen, sondern kontrafaktisch sind, hängt es - auch - von unseren Werthaltungen ab, wie groß wir uns die verhinderten Übel vorstellen. Da sind wir wieder bei den rätselhaften Wenn/ dann-Sätzen, von denen vorhin die Rede war: Wenn wir den Krieg nicht geführt hätten, dann wäre das und das geschehen. Objektiv kennt das, was geschehen wäre, kein Mensch - der pazifistische Anhänger der Lehre vom Gerechten Krieg genausowenig wie der bellizistische Anhänger dieser Lehre.

Betrachten wir zweitens die Rede vom äußersten Notfall (ultima ratio). Sie gehört zum ius ad bellum, also zum Katalog der Kriterien, die zum Kriegsbeginn berechtigen. Nur wenn nichts anderes übrigbleibt, so die Idee, nur dann darf man gegen das zu verhindernde Übel mit den Mitteln des Kriegs einschreiten. ${ }^{16}$ Das kann der Pazifist locker unterschreiben. Anders als die Kriegsbefürworterin wird er so gut wie immer der Ansicht sein, dass es doch noch unkriegerische Mittel gibt, mit denen man es versuchen sollte. Seine Kontrahentin wird das bestreiten, aber durch objektiven Blick auf die Fakten lässt sich auch dieser Streit nicht schlichten; es ist u.a. eine Frage der Phantasie. Beide Streitparteien blicken im Lichte unterschiedlicher Werthaltungen auf die Fakten. Beide - also nicht nur der Pazifist. Und was ich eben für zwei Kriterien aus der Lehre vom Gerechten Krieg vorgeführt habe, gilt allgemein. Bereits bevor man irgendwelche moralischen Kriterien auf Krieg und Frieden anwenden will, müssen Werte herangezogen werden. Welche Werte? Das hängt davon ab, ob man Pazifist ist oder nicht.

Kurzum, um Pazifist zu sein, kann und muss ich mehr sagen als: „Krieg ist immer moralisch verboten, Ende der Durchsage“. Die hinter diesem Satz stehende Haltung wird zwar ebenfalls als Pazifismus bezeichnet; sie ist aber zu bequem. Ich plädiere also für eine anstrengende Form von Pazifismus. Man muss viel Selbstdisziplin aufbieten, um auf dem Boden der Tatsachen zu bleiben und von dort aus seriös (bei vollem Bewusstsein der hinzukommenden Werthaltungen) gegen unsere Kriege zu argumentieren. Der erkenntnistheoretische Pazifist wird beispielsweise dafür werben, dass seine friedliebende Betrachtung der Situation im Vorfeld eines geplanten Kriegs attraktiver ist als das Wertsystem, unter dem seine Kontrahenten dieselbe Situation betrachten. Er wird dem pessimistischen Menschenbild seiner Kontrahenten widersprechen und ihren Optimismus für angeblich kalkulierbare Folgen von Waffenschlägen für naiv erklären. Er plädiert für die umgekehrte Kombination aus Optimismus und Pessimismus: Pessimismus zur militärisch-technischen Kontrollierbarkeit von Konflikten plus optimistisches Menschenbild (demzufolge gewaltbereite Menschen nicht immer nur mit vorgehaltener Knarre zur Raison gebracht werden müssen).

Das ist ein Streit über die angemessenen Bezugspunkte von Optimismus und Pessimismus, den uns die objektiven Fakten nicht abnehmen; wir müssen selber entscheiden. Und da frage ich Sie: Wären Sie lieber Optimist über gewaltfreie oder über gewalttätige Ansätze in der Politik? Oft höre ich von meinen Gegnern: Es wäre ja schön, wenn Du recht hättest, aber die Welt ist nicht so. Dass die letzten fünf Worte dieser Erwiderung keinen Realismus ausdrücken, steht im Lichte meiner metaethischen Thesen

16 Hierzu und zum Folgenden siehe O.M. [PmOA], 9. Abschnitt. aus Abschnitten 4 und 5 fest; es sind fünf pessimistische Worte. Und sobald jemand erkannt hat, dass er Realismus mit Pessimismus verwechselt hat und dass pessimistische Menschenbilder unschön sind (nach seiner eigenen Aussage!), kann er dem pazifistischen Blick auf die Fakten vielleicht mehr abgewinnen. In diesen Blick gehen Tugenden ein wie Behutsamkeit, Sorge vor Selbstüberschätzung, Hoffnung auf das friedfertige Potenzial in jedem Menschen, Phantasie und Menschenliebe. ${ }^{17}$

\section{Mutmaßungen über Mali}

Zum Abschluss meiner Betrachtung möchte ich das Gesagte auf die französische Intervention in Mali anwenden. Wie bislang in diesem Aufsatz werde ich auch hier nicht tief genug in die vielen wichtigen Details eindringen; doch anders als bislang kann ich jetzt nicht wieder auf Veröffentlichungen anderswo verweisen, in denen die Details zu finden sind. Der Grund: Ich weiß zu wenig über diesen Krieg. Und so werde ich nur einige Andeutungen machen, die aus meiner Sicht etwas Wasser auf die Mühlen des Pazifismus fließen lassen. Dabei stütze ich mich auf Faktenbehauptungen aus der Frankfurter Allgemeinen Zeitung. In einer vertieften ethischen Analyse wären alle dort angeführten Fakten eigenhändig zu überprüfen, und das kann ich hier nicht leisten.

Ist es unzulässig, dass ich mich auf ungeprüfte Faktenbehauptungen stützen werde? Nein, und zwar aus folgendem Grund. Die FAZ hat den französischen Krieg in Mali von Anbeginn für richtig und zulässig erklärt. ${ }^{18}$ Und nach den Regeln der Logik darf man sich beim Argumentieren immer auf die Voraussetzungen des Kontrahenten stützen, um ihm einen Fehler nachzuweisen. Genau das will ich jetzt versuchen.

Doch bevor ich damit anfange, möchte ich eine weitere Vorbemerkung voranschicken: Die Ziele der französischen Intervention teile ich allesamt. Ich finde es empörend und gruselig, wenn Islamisten ein ganzes Land in ihren Würgegriff nehmen wollen und so weit gehen, die Weltmusik einer grandiosen Sängerin wie Rokia Traoré zu verbieten. ${ }^{19}$ Und selbstverständlich leiden nicht nur Prominente unter dem haarsträubenden Gehabe der Islamisten; für die Frau auf der Straße dürfte es noch weit schlimmer kommen - falls sie sich dann überhaupt noch auf die Straße traut. Kurzum, ich kann den Impuls, sofort einzuschreiten, gut nachvollziehen. Und ich bin mir bewusst, dass der Verzicht darauf so hätte verstanden werden können, als ließen wir Europäer eine ganze Kultur im Stich. Schuldfrei und mit reiner Weste stünden wir danach nicht da. Alles das gebe ich zu. Und dennoch fürchte ich, dass es infolge der Intervention für viele einzelne Menschen noch viel schlimmer kommen wird. Ich fürchte es dass ich es wüsste, werde ich als erkenntnistheoretischer Pazifist nicht behaupten. Ich habe zwei Einwände gegen den Mali-Krieg.

\subsection{Erster Einwand: ius post bellum}

Meinen ersten Einwand (aber nicht den zweiten) formuliere ich im Rahmen der Theorie des Gerechten Kriegs. Diese Theo-

17 Siehe O.M. [PmOA], 12. Abschnitt und O.M. [WWSü], 9. Abschnitt.

19 Siehe FAZ, 22.1.2013, S. 31. 
rie ist in den letzten Jahren sehr sorgfältig entfaltet und ausgebaut worden - von Denkern und Denkerinnen wie Michael Walzer und Jean Bethke Elshtain, die sich immer wieder für die prinzipielle Berechtigung von Krieg ausgesprochen haben. ${ }^{20}$ Eine wichtige Innovation ihrer ethischen Forschungen ist das sog. ius post bellum. ${ }^{21}$ Nicht nur gilt es, so die Idee, Bedingungen für Beginn und Durchführung eines ethisch korrekten Kriegs zu beachten (das sind die schon erwähnten Kriterien von ius ad bellum und ius in bello); vielmehr ist zusätzlich darauf zu achten, dass der Intervenierende auch nach Kriegsende alles Erforderliche tut, um die von ihm geschaffene Situation moralisch akzeptabel werden zu lassen - für alle Seiten des Konflikts, in den er eingegriffen hat.

Hier ist einer der vielen guten Gründe, die für diese Ausweitung und Ergänzung der Theorie sprechen: Wer aus Verantwortungsbewusstsein in einen Konflikt eingreift, ist für alle Folgen seines Tuns verantwortlich, auch für die Spätfolgen, und kann sich aus dieser Verantwortung nicht einfach mit dem Spruch mission accomplished davonstehlen.

Genau das aber plant der französische Präsident - jedenfalls nach allem, was er dazu am Anfang gesagt hat. ${ }^{22}$ Vielleicht hat er Glück, und das Land kommt nach der Intervention wieder von selbst auf die Beine, ohne dass die Günstlinge der Franzosen alte Rechnungen begleichen oder deren Gegner eine Revanche anzetteln. ${ }^{23}$ Aber es gehört schon eine gehörige Prise Selbstvertrauen dazu, auf dies Glück zu bauen. Man könnte es auch als Vabanquespiel bezeichnen. ${ }^{24}$ Erkenntnistheoretische Pazifisten werden bestreiten, dass der französische Kriegsherr oder seine Berater vorab sorgfältig genug eruieren konnten, womit nach Kriegsende zu rechnen ist. ${ }^{25}$ Ich füge hinzu: Hoffentlich stimmt das nicht. Und: Hoffentlich geht die französische Wette auf.

20 Walzer [JUW], Elshtain [JWaT].

21 Elshtain [WPiT] und Walzer [AaHI], S. 30-32, insbes. S. 31. Diese Innovation ist keine reine Erfindung irgendwelcher Intellektueller; sie ist von hochrangigen amerikanischen Militärs und Politikern auf den Tisch gelegt worden, und zwar unter der Überschrift Weinberger/Powell-Doktrin (so jedenfalls der ehemalige Staatssekretär im deutschen Verteidigungsministerium Hans Rühle in einem Leserbrief, siehe FAZ, 30.1.2013, S. 29).

22 FAZ, 17.1.2013, S. 1/2, FAZ, 30.1.2013, S. 5. Siehe aber FAZ, 27.2.2013, S. 6

23 Ich nenne nur einige beunruhigende Indizien für die erste Gefahr, die ich allesamt der FAZ entnommen habe. So gab ein prominenter Tuareg im Januar 2013 zu Protokoll: „Ich habe das dumme Gefühl, dass wir Tuareg einen hohen Preis zahlen werden, wenn dieser Spuk vorbei ist" (FAZ, 22.1.2013, S. 8). Erste Berichte für Lynchjustiz durch Soldaten der malischen Armee, auf deren Seite die Franzosen stehen, liefert die FAZ, 25.1.2013, S. 2. Dass die Gefahr ernstgenommen werden sollte, haben die Franzosen schnell bemerkt: Weil sich die Tuareg in Midal vor Übergriffen malischer Truppen fürchteten, wurde die Stadt nach ihrer Übergabe vernünftigerweise nicht von Soldaten aus Mali, sondern von Soldaten aus dem Tschad kontrolliert (FAZ, 6.2.2013, S. 1/2). Ich frage: Wer soll nach Abzug der Franzosen für solche vernünftigen Regelungen sorgen?

24 Nicht ganz so undiplomatisch hatten dieselbe Sorge zwei französische Spitzendiplomaten ausgedrückt, bevor sie wegen ihrer skeptischen Haltung (etwa zu den Chancen eines demokratischen Wiederaufbaus in Mali) ihrer Zuständigkeit enthoben worden sind (FAZ, 22.3.2013, S. 6).

25 Dazu drei beunruhigende Indizien. Erstes Indiz: Wie aussichtslos es erscheint, die gegenwärtige Tuareg-Frage mal eben mit links zu lösen, ergibt sich z.B. aus der gerafften Darstellung ihrer Vorgeschichte, siehe FAZ, 19.1.2013, S. 4. Zweites Indiz: Am 8.2.2013 haben sich zwei zerstrittene Soldatengruppen der malischen Armee in Bamako eine Schießerei geliefert (FAZ, 9.2.2013, S. 1/2). Ebenso wie der Beginn der Selbstmordattentate zeigt dies, dass die französische Armee in Mali mehr leisten muss, als einfach nur den Vormarsch der Dschihadisten zu stoppen (so FAZ, 9.2.2013, S. 10). Drittes Indiz: Der naheliegende Plan, sich post bellum durch Einrichtung einer Blauhelmtruppe aus der Affäre zu ziehen, birgt eigene Gefahren; so weist die FAZ mit Recht darauf hin, dass Soldaten der UN-Friedenstruppen immer wieder die strengen menschenrechtlichen Standards verletzt haben, die für sie gelten (FAZ, 8.2.2013, S. 6). Das ist eine zurückhaltende Beschreibung, siehe z.B. FAZ, 10.1.2005, S. 2.
Dass man daran zweifeln kann, haben die Erfahrungen der letzten Kriege gelehrt; besonders verstörende und bittere Lektionen hat uns die Entwicklung in Afghanistan zugemutet. ${ }^{26}$ Der Westen zieht sich von dort ja nicht deshalb zurück, weil beruhigenderweise feststünde, was nach unserem Abzug geschehen wird - sondern trotz größter Ungewissheit. Und man muss kein Gedankenleser sein, um zu vermuten, dass gerade das afghanische Abenteuer den französischen Präsidenten dazu bewogen hat, von vornherein auf die Beachtung der Regeln des ius post bellum verzichten zu wollen. ${ }^{27}$

Das Geschäft der Moral ist kein Selbstbedienungsladen; Rosinenpickerei gilt nicht. Obwohl ich Pazifist bin oder gerade weil ich es bin, stimme ich den Anhängerinnen und Anhängern der Theorie des Gerechten Kriegs zu: Wer sich nicht zutraut, nach Kriegsende die durch den Krieg zerbrochene (oder jedenfalls durch ihn weiter erodierte) Ordnung zu reparieren, darf den fraglichen Krieg gar nicht erst anfangen. Und wer sich's zutraut, ohne darin gerechtfertigt zu sein, muss ebenfalls auf Kriegseintritt verzichten. Genauso bei demjenigen, der ungerechtfertigterweise darauf baut, dass nach Kriegsende alles von allein wieder in Ordnung kommt. Korrekte Kriegführung verlangt hohe Standards, auch mit Blick auf Wissen und erkenntnistheoretische Sorgfalt; wem diese Standards zu hoch sind, der darf die Latte nicht eigenmächtig tiefer hängen.

Oder hängt die Latte etwa grundsätzlich zu hoch? Steht von vornherein fest, dass man sie gar nicht überspringen kann? Nein; das zeigen das Ende des Zweiten Weltkriegs und das Schicksal derjenigen Zonen Deutschlands, für die damals Amerikaner, Briten und Franzosen verantwortlich waren: Die westlichen Siegermächte halfen dabei, ein am Boden liegendes Land wiederaufzubauen - ökonomisch, politisch, juristisch, sogar halbwegs moralisch. Warum sie das taten, ist für meine Zwecke gleichgültig. Vielleicht verfolgten sie allerlei eigennützige Zwecke. Aber sie taten es. Der Krieg der westlichen Alliierten gegen Nazi-Deutschland hat die Bedingungen des ius post bellum verblüffend gut erfüllt. Es kann also gehen.

\subsection{Zweiter Einwand: Die Spätfolgen des einen Kriegs sind die Vorgeschichte des andern}

Krieg ist eine ansteckende Krankheit. In diesem Punkt denkt der erkenntnistheoretische Pazifist pessimistisch. ${ }^{28}$ Nicht, dass er wüsste, wie ansteckend diese Krankheit in jedem einzelnen Fall sein wird - er befürchtet nur die Weiterverbreitung des bellizistischen Bazillus. Und die Geschichte der letzten Jahrhunderte nährt diese Befürchtung. Daher empfiehlt er, die

26 Siehe z.B. FAZ, 14.1.2013, S. 1. Die Entscheidungsträger scheinen gute Miene zum bösen Spiel machen und ihr Gesicht wahren zu wollen, indem sie im sog. Fortschrittsbericht von Erfolgen erzählen, wo keine sind (siehe z.B. FAZ, 5.2.2013, S. 8).

27 Ähnlich (ohne ausdrücklichen Verweis aufs ius post bellum) FAZ, 4.2.2013, S. 1.

28 Während die Opponenten des Pazifisten auf ihre starken Nerven stolz sind, kultiviert der Pazifist eine pessimistische Haltung überall da, wo er neue Kriegsgefahren wittert; er wirft seinen optimistischen Opponenten Blauäugigkeit und mangelnde Sensibilität für Kriegsgefahren vor. Wer in dieser Auseinandersetzung recht hat, steht nicht objektiv fest - es ist auch eine Frage der Wertung; hier haben wir wieder ein Beispiel für die unentwirrbare Durchdringung von Werten und Fakten. 
Kette aus Gewalt und Gegengewalt zu unterbrechen, notfalls einseitig und sogar dann, wenn Eingreifen geboten scheint.

Kann er seine Empfehlung nur mit geschlossenen Augen aufrechterhalten, also am Ende blind für die Umstände, die schnelles Eingreifen erheischen, wie z.B. im Januar 2013 in Mali? Nein, ganz im Gegenteil - als Pessimist öffnet er seine Augen und versucht, weiter zu schauen als seine Kontrahenten. Er nennt sie kurzsichtig, und dafür hat er folgenden guten Grund.

Oft genug in letzter Zeit hat sich die martialische Strategie schließlich gegen diejenigen gewendet, die sie eingesetzt haben. Der Westen hat die afghanischen Freiheitskämpfer gegen die UdSSR bewaffnet und trainiert; später kämpfte er gegen sie. Er hat den Irak im Krieg gegen den Iran mit Waffen versorgt; später führte er gegen den so ausgerüsteten Diktator Krieg, und das zweimal. Die Kette solcher Beispiele setzt sich bis in die Gegenwart fort, bis hin zu den vorläufig letzten Gliedern. Sie haben unmittelbar mit der Vorgeschichte des Kriegs in Mali zu tun. ${ }^{29}$

Um das zu demonstrieren, frage ich: Woher kommt die Tuareg-Bewegung für die Befreiung des Azawad (MNLA), die sich mit Dschihadisten zusammengetan hat, weil sie auf diese Weise einen unabhängigen Staat für die Tuareg glaubte erreichen zu können? Antwort aus der FAZ: Diese Rebellen haben zuvor in der libyschen Armee gedient und sich „nach Gaddafis Tod unter Mitnahme von schweren Waffen nach Mali abgesetzt". ${ }^{30}$

Hier sehen wir also eine Spätfolge der hochgelobten LibyenIntervention, die zur Vorgeschichte des Mali-Kriegs geworden ist. Dessen Befürworter Thomas Scheen schrieb dazu:

„Das kann man ebenso beklagen wie das forsche Vorgehen des damaligen Präsidenten Sarkozy gegen den libyschen Diktator Gaddafi, ohne das der Sahel heute vermutlich nicht der Waffenbasar wäre, der er geworden ist. Nur nützt dieses Lamentieren angesichts der gegenwärtigen Lage niemandem. Dafür ist das Bedrohungspotential, das von den Islamisten in Mali ausgeht, einfach zu groß geworden. Die neue französische Regierung hat das erkannt und konsequent gehandelt. Die Frage, ob die Präsenz weißer Soldaten in Mali nunmehr die Internationale der Islamisten anziehen wird wie die Motten das Licht und der Konflikt folglich eskaliert, stellt sich aber schon deshalb nicht, weil die längst da sind [...] Diese Gegner sind ein paar Nummern zu groß für die Afrikaner; sie mit ihnen alleinzulassen käme den Westen langfristig teurer zu stehen als die ,Opération Serval'." (FAZ, 29.1.2013, S. 1; Hervorheb. O.M.)

Dazu zwei Fragen. Erstens, woher nimmt der Autor die Begründung für seinen letzten Satz? Er kommt wie eine kühl abgewogene, objektive Wahrheit daher, zeigt aber vielleicht nur die Phantasielosigkeit des Autors und sein martialisches Wertsystem. Der Satz steht im Konjunktiv und erinnert an die rätselhaften Wenn/dann-Sätze, von denen im 5. Abschnitt die Rede war. Auch die kursiv hervorgehobene Behauptung vom Beginn des Zitats kann keine Objektivität für sich bean-

29 Dass auch in diesem Krieg schon das übernächste Glied jener Kette geschmiedet werden könnte, befürchtete der ägyptische Präsident Muhammad Mursi (zitiert nach FAZ, 23.1.2013, S. 2).

30 FAZ, 22.1.2013, S. 8, Hervorheb. O.M. Ähnlich FAZ, 19.1.2013, S. 4. spruchen; auch sie steht im Konjunktiv und läuft auf einen kontrafaktischen Wenn/dann-Satz hinaus. Wie Sie gleich sehen werden, stütze ich mich dennoch auf diesen Satz; es ist (wie gesagt) immer erlaubt, mit den Annahmen des Gegners weiterzuargumentieren.

Zweitens, warum sollen wir immer so weitermachen wie bislang? Vor allem dann, wenn eben diese Vorgehensweise kürzlich einen neuen Waffenbasar geschaffen hat? Gibt es nicht zu denken, wenn die Spätfolgen des einen Kriegs kurz darauf als Vorgeschichte des nächsten Kriegs wieder hochkommen? Aus der Rückschau ist man immer schlauer; aber warum versuchen wir nicht, das Gelernte in die Zukunft zu übertragen? Die Lektion ist doch bereits oft genug gelernt worden. Noch in derselben Ausgabe der FAZ konnte man lesen, dass in Frankreich strukturell ähnliche Diagnosen zur Vorgeschichte der malischen Misere kursierten, mit Blick auf die Rolle der USA:

„Es mehren sich französische Presseberichte, in denen Amerika mit zur Verantwortung gezogen wird für das Erstarken der Terrorgruppen in Mali. Es wird darauf hingewiesen, dass drei der vier von amerikanischen Militärausbildern geschulten und mit modernstem amerikanischem Material ausgerüsteten malischen Eliteeinheiten im Frühjahr 2012 zu den Terrorgruppen übergelaufen waren. Zudem wird daran erinnert, dass die Schwächung des malischen Staates auf einen in Amerika ausgebildeten Hauptmann, Amadou Sanogo, zurückgeht, der 2012 einen Putsch organisierte und damit den Vormarsch der Terrorgruppen ermöglichte." (FAZ, 29.1.2013, S. 2)

Die eingangs im Zitat erwähnten Presseberichte waren nicht aus der Luft gegriffen. ${ }^{31}$ Eine Woche später hieß es im Gespräch des französischen Verteidigungsministers Jean-Yves Le Drian mit der Jounalistin Michaela Wiegel:

\section{„Die amerikanische Armee hatte Milliardenbeträge in die Ausbil- dung malischer Offiziere investiert, viele von ihnen jedoch schlos- sen sich der Rebellion an. Wie verhindern Sie einen ähnlichen Fehlschlag?}

Aus der bedauerlichen amerikanischen Erfahrung haben wir vor allem gelernt, dass staatliche Einheit und Einheit der Armee nicht voneinander zu trennen sind. Die Armee muss das gesamte Staatsgebilde widerspiegeln." (FAZ, 6.2.2013, S. 2)

Was der französische Verteidigungsminister hier zu Protokoll gibt, läuft letztlich auf folgende Naivität hinaus: Gut, zugegeben, früher hat man allerlei Fehler gemacht und dadurch unerwünschte langfristige Kriegsgefahren heraufbeschworen, aber erstens waren das die andern, und zweitens ist diesmal alles anders. Lassen Sie diese paar Splitter auf sich wirken. Was meinen Sie: Wollen wir's nicht zur Abwechslung einmal ohne Waffen versuchen?

31 Schon vorher stand in der FAZ ein schockierender Bericht über die Fehler, die von den Amerikanern im Mali gemacht wurden und die das dortige Problem erst geschaffen haben (FAZ, 17.1.2013, S. 2). 


\section{Optimistischer Ausblick}

Vielleicht wird jemand einwenden, ich hätte Eulen nach Athen getragen. Immerhin setzt sich unsere bürgerliche Regierung gegen Waffenlieferungen an die syrische Opposition ein, und sie hat sich weder an den Kriegen in Libyen noch in Mali beteiligt. Für mich ist das ein Hoffnungsstreifen am Horizont. Möglicherweise steckt unseren augenblicklichen Entscheidungsträgerinnen der Schock vom afghanischen Abenteuer noch in den Knochen. Aber warum sagen sie das nicht ausdrücklich? Sicher, es ist nobel, wenn sie die Schuld nicht einfach auf die rot-grüne Vorgängerregierung abschieben, die sich in das Abenteuer gestürzt hat. Doch wäre es besser, wenn wir erführen, worauf die neue deutsche Vorsicht beruht. Hoffentlich bleibt sie uns erhalten; und hoffentlich wirkt sie am Ende ansteckend auf unsere Verbündeten. ${ }^{32}$

\section{Literatur}

Bleisch, Barbara / Strub, Jean-Daniel (eds.) [PITP]: Pazifismus Ideengeschichte, Theorie und Praxis. (Bern: Verlag Haupt, 2006).

Elshtain, Jean Bethke [JWaT]: Just war against terror. The burden of American power in a violent world. (New York: Basic Books, 2003).

Elshtain, Jean Bethke [WPiT]: „War and peace in the 21st century - new challenges and normative consequences“. In Strub et al. (eds.) [GFzP]: S. 263-270.

Fehige, Christoph / Meggle, Georg (eds.) [zMD]/1: Zum moralischen Denken. Band 1. (Frankfurt: Suhrkamp, 1995).

Löwenstein, Stephan [DIEG]: „,Deutschland ist erwachsener geworden'. Verteidigungsminister Struck über sieben Jahre rot-grüner Verteidigungspolitik“. (Interview). Frankfurter Allgemeine Zeitung (26.8.2005), S. 6.

McDowell, John [AMRH]: „Are moral requirements hypothetical imperatives?" Proceedings of the Aristotelian Society. Supplementary Volume LII (1978), S. 12-29.

Meggle, Georg (ed.) [EoHI]: Ethics of humanitarian interventions. (Frankfurt/Lancaster: Ontos Verlag, 2004).

Meggle, Georg (ed.) [HI]: Humanitäre Interventionsethik. Was lehrt uns der Kosovo-Krieg? (Paderborn: Mentis, 2004).

Millgram, Elijah [IEB]: „Inhaltsreiche ethische Begriffe und die Unterscheidung zwischen Tatsachen und Werten“. In Fehige et al. (eds.) [zMD]/1: S. 354-388.

Müller, Olaf $[\mathrm{CKK}]:$ „Chaos, Krieg und Kontrafakten. Ein erkenntnistheoretischer Versuch gegen die humanitären Kriege“. In Bleisch et al. (eds.) [PITP]: S. 223-263. [Im Netz unter http://nbn-resolving.de/urn:nbn:de:kobv:11-100187305].

32 Ich danke Sabine Jaberg und Benjamin Marschall für hilfreiche Verbesserungsvorschläge zu einer früheren Fassung dieses Aufsatzes. Benjamin Marschall hat mich dankenswerterweise bei der Arbeit an der Bibliographie, bei der Endredaktion und bei einer Reihe von Recherchen unterstützt. Er legt Wert auf die Feststellung, dass er die Kernthesen meines Ansatzes nicht teilt; umso größer mein Dank für die Zusammenarbeit. Dank an Katharina Nagel für die Überprüfung der wörtlichen Zitate.
Müller, Olaf [HPAS]: Hilary Putnam und der Abschied vom Skeptizismus oder Warum die Welt keine Computersimulation ist. (Paderborn: Mentis, 2003). [Eine Kurzfassung im Netz unter http://nbn-resolving.de/urn:nbn:de:kobv:11-100187495].

Müller, Olaf [PmOA]: „Pazifismus mit offenen Augen“. In Strub et al. (eds.) [GFzP]: S. 23-59. [Im Netz unter http://nbn-resolving.de/urn:nbn:de:kobv:11-100187305].

Müller, Olaf [PP]: Philosophie des Pazifismus. Fallstudien und ethische Argumente gegen den Krieg. (Hamburg: tredition, 2013). [Als E-Buch und auf Papier; im Erscheinen].

Müller, Olaf [UG]: „Unwegsames Gelände. Pazifistische Notrufe zu Afghanistan“. epd Dokumentation 13/14 (2011), S. 18-25.

Müller, Olaf [WWSü]: „Was wissen Sie über Kosovo? - Fallstudie über Pazifismus, Propaganda und die Verquickung von Fakten mit Werten“. In Meggle (ed.) [HI]: S. 53-90. [Im Netz unter http://nbn-resolving.de/urn:nbn:de:kobv:11-10066420].

Murdoch, Iris [IoP]: „The idea of perfection“. In Murdoch [SoG]: S. 1-45.

Murdoch, Iris [SoG]: The sovereignty of good. (London: Routledge $\&$ Kegan Paul, 1970).

Putnam, Hilary [CoFV]: The collapse of the fact/value dichotomy and other essays. (Cambridge / Mass.: Harvard University Press, 2002).

Putnam, Hilary [OSED]: „Objectivity and the science/ethics distinction“. In Putnam [RwHF]: S. 163-178.

Putnam, Hilary [RTH]: Reason, truth and history. (Cambridge: Cambridge UP, 1981).

Putnam, Hilary [RwHF]: Realism with a human face. (James Conant (ed.); Cambridge / Mass.: Harvard University Press, 1990).

Rickert, Heinrich [KN]: Kulturwissenschaft und Naturwissenschaft. (Tübingen: Mohr 1910). [Erschien zuerst 1899].

Strub, Jean-Daniel / Grotefeld, Stefan (eds.) [GFzP]: Der gerechte Friede zwischen Pazifismus und gerechtem Krieg. Paradigmen der Friedensethik im Diskurs. (Stuttgart: Kohlhammer, 2007).

Walzer, Michael $[\mathrm{AaHI}]:$ „The argument about humanitarian intervention“. In Meggle [EoHI]: S. 21-35.

Walzer, Michael [JUW]: Just and unjust wars. (New York: Basic Books, 1977).

Williams, Bernard [ELoP]: Ethics and the limits of philosophy. (Cambridge / Mass.: Harvard University Press, 1985). 